\title{
Prioritizing Consumers in Smart Grid: Energy Management Using Game Theory
}

\author{
Wayes Tushar*§, Jian A. Zhang ${ }^{\ddagger}$, David B. Smith ${ }^{\S *}$, Sylvie Thiebaux ${ }^{\S *}$ and H. Vincent Poor $^{\dagger}$ \\ ${ }^{*}$ College of Engineering and Computer Science, Australian National University, ACT, Australia. Email: wayes.tushar@anu.edu.au \\ $\ddagger$ CSIRO ICT Center, Marsfield, NSW, Australia. Email: andrew.zhang@csiro.au \\ $\S$ National ICT Australia (NICTA), Canberra, ACT, Australia. Email: \{david.smith, sylvie.thiebaux $@ @$ nicta.com.au \\ ${ }^{\dagger}$ School of Engineering and Applied Science, Princeton University, Princeton, NJ, USA. Email: poor@princeton.edu
}

\begin{abstract}
This paper explores an idea of demand-supply balance for smart grids in which consumers are expected to play a significant role. The main objective is to motivate the consumer, by maximizing their benefit both as a seller and a buyer, to trade their surplus energy with the grid so as to balance the demand at the peak hour. To that end, a Stackelberg game is proposed to capture the interactions between the grid and consumers, and it is shown analytically that optimal energy trading parameters that maximize customers' utilities are obtained at the solution of the game. A novel distributed algorithm is proposed to reach the optimal solution of the game, and numerical examples are used to assess the properties and effectiveness of the proposed approach.

Index Terms-Smart grid, two-way communication, demand management, Stackelberg game, consumer's benefit, variational equilibrium.
\end{abstract}

\section{INTRODUCTION}

The smart grid (SG) is envisioned to be a large-scale next generation cyber-physical system that will improve the efficiency, reliability, and robustness of future power and energy grids by integrating the consumers as one of its key management components [1], and thus, achieve a system which is clean, safe, reliable, resilient and sustainable. This heterogeneous network will motivate the adoption of advanced technologies that will increase the participation of its consumers to overcome various technical challenges at different levels of demand-supply balance [1].

In this respect, game theory, which is an analytical framework to capture the complex interactions among rational players [2] is studied in this paper to model an energy trading scheme for the SG. The model uses the two-way communication facility of the SG [3], and inspires the customers to spontaneously take part in supplying their surplus energy (SE) to the grid so as to assist the power grid (PG) in balancing the excess energy demand at the peak hour. This voluntary participation of consumers in energy trading is very important in the context of SG because of its ability to greatly enhance the SG's reliability, and thus, improve the social benefit of the

This work is supported by NICTA. NICTA is funded by the Australian Government as represented by the Department of Broadband, Communications and the Digital Economy and the Australian Research Council through the ICT Centre of Excellence program. This work is also supported in part by the U. S. Air Force Office of Scientific Research under MURI Grant FA9550-09 $1-0643$ electricity market [4]. We use the framework of a Stackelberg game [2] for this model in which the PG is considered as the leader and energy users (EUs) are the followers. Here, on the one hand, the PG decides on the total amount of energy it wants to buy, and also on the price per unit of energy it needs to pay to each EU. On the other hand, the EU decides on its amount of energy to be sold to the PG in response to the price offered to it.

We note that energy management in the context of SG has been receiving considerable attention recently. For example, energy management for SGs in a vehicle-to-grid (V2G) scenario have been studied in $[1,3,5]$ and the references therein, whereby the application of game theory for demandsupply balance in SGs can be found in [3] and [5]. However, little has been done in prioritizing the consumers' benefit in management modeling where the main priority of the energy management scheme is to benefit the consumers. We stress that consumers are the core element of the evolution of SG as explained in [6], and hence, their benefit is one of the most important concerns of any demand-supply modeling scheme. In this respect, we propose an energy management scheme that prioritizes the consumers in the SG and balances demand with supply at peak hours. We first formulate a noncooperative Stackelberg game (NSG) to study the interactions between the PG and EUs in the SG, and show that the optimal demand-supply balance can be achieved at the solution of the game; then we analyze properties of the game in terms of existence and optimality, and it is shown that the game possesses a socially optimal solution; finally, we propose a distributed algorithm to reach the solution of the game, and the effectiveness of the proposed scheme is demonstrated via numerical experiments.

\section{System Model}

Consider an SG network that consists of a single PG and a number of EUs. The set of EUs is $\mathcal{N}$, where $|\mathcal{N}|=N$. Here, the PG refers to the main electricity grid which is servicing a group of customers at peak hours of the day (i.e., $12 \mathrm{pm}$ to $4 \mathrm{pm}$ ), and each EU $i \in \mathcal{N}$ is a group of similar idle energy users [1], connected via an aggregator [7], such as smart homes, electric vehicles, wind mills, solar panels and 
bio-gas plants that have some SE for sale after regular usage. It is assumed that the PG can communicate with the EUs through smart meters via an appropriate communication protocol [3].

Due to frequent change of energy state in the grid, energy management in the SG needs to be carried out frequently [5] and therefore, the total peak hour duration can be divided into multiple time slots [5]. As energy demands by the customers are very high during the peak hours, the PG may be unable to balance some of the demands from its own generation in some of these time slots. Meanwhile, the PG needs to buy energy from alternative energy sources such as idle EUs who have SE, and may agree to sell it to the PG with appropriate incentives. For the rest of the paper, we will concentrate on the energy management in a single time slot. It is assumed that the energy deficiency of the PG, $E_{\mathrm{PG}}$, at any time slot is fixed although the deficiency may vary from one time slot to the next. However, as the required energy by the PG is fixed during a time slot, the PG would not be interested in buying more energy than $E_{\mathrm{PG}}$ to keep its cost at a minimum. Thus, if each EU $i$ with SE $E_{i}$ provides the PG with energy $x_{i}$, these quantities need to satisfy

$$
\sum_{i} x_{i} \leq E_{\mathrm{PG}} ; x_{i} \leq E_{i}, \forall i .
$$

To buy an offered amount of energy $x_{i}$, the PG pays a price $p_{i}$ per unit of energy to EU $i$ as an incentive. However, the PG may need to pay different incentives to different EUs due to their different amounts of SE. For instance, a lower incentive may not affect the intended revenue of an EU with higher SE as it can sell more, but could severely affect the revenue of EUs with smaller amounts of SE. Moreover, the PG may also want to minimize its total cost of purchase as it would further enable the PG to sell this energy at a cheaper rate to its customer. This would facilitate the trading of energy between the PG and the EUs in the network rather than establishing more expensive generators or bulk capacitors to meet any excess demand, and also, the cheaper rate would benefit the consumers who buy the energy from the PG. To this end, we assume that the PG estimates a total price $P_{r}$ per unit of energy, analogous to the total cost per unit production in economics [8], in each time slot using a real time price estimation technique as proposed in [9]. The PG uses this $P_{r}$ to optimize the price $p_{i}$ it will pay to each EU $i$ to order to minimize its total cost while maintaining the constraint

$$
\sum_{i} p_{i}=P_{r} ; p^{\min } \leq p_{i} \leq p^{\max } .
$$

The equality constraint in (2) establishes that the announced total price per unit energy must be paid to all EUs, and thus motivates the EUs to take part in energy trading with the PG. Here, $p^{\min }$ is the minimum price that the PG needs to pay EU $i$ to incentivize it to trade energy, and $p^{\max }$ is the maximum price that the PG can pay. Although $P_{r}$ is fixed, $p_{i}$ can be different for different $i$ based on $x_{i}$.

\section{Stackelberg Game And Its Properties}

In a consumer-prioritized SG, the beneficiaries of the energy management scheme are the consumers in the network [6]. In this regard, we propose an NSG, in which on the one hand, the objective of each EU $i$ is to voluntarily sell an amount of energy $x_{i}$ to the PG based on $E_{i}$ and the offered price $p_{i}$ so as to maximize its own utility. On the other hand, the PG wants to minimize its total cost of purchase by optimizing $p_{i}$ for different $i$ as explained in Section II. To this end, we now define the objective functions of the leader and followers of the game.

\section{A. Objective functions of the EUs and the $P G$}

The considered utility function of $\mathrm{EU} i, U\left(x_{i}, E_{i}, p_{i}\right)$, is based on a linearly decreasing marginal benefit, which is appropriate for energy users [10]. In addition, the utility function is also assumed to possess the following properties: i) the utility of EU $i$ increases with the amount of SE $E_{i}$, i.e., $\frac{\delta U}{\delta E_{i}}>0$; and ii) the utility of an EU increases as $p_{i}$ increases, i.e., $\frac{\delta U}{\delta p_{i}}>0$. To meet the above properties, in this work we consider the following utility function for $\mathrm{EU} i$ :

$$
U\left(x_{i}, E_{i}, p_{i}\right)=E_{i} x_{i}-\frac{1}{2} x_{i}{ }^{2}+p_{i} x_{i} .
$$

From (3), we note that the addition of the quantity $\sum_{j \neq i} E_{j} x_{j}-\frac{1}{2} x_{j}{ }^{2}+p_{j} x_{j}$ to $U$ in (3) does not affect the solution. Consequently, all the EUs equivalently maximize

$$
\hat{U}(\mathbf{x}, \mathbf{E}, \mathbf{p})=\sum_{i=1}^{N} U\left(x_{i}, E_{i}, p_{i}\right),
$$

subject to the constraint $\sum_{i} x_{i} \leq E_{\mathrm{PG}}, \forall i$. Here, $\mathbf{x}=$ $\left[x_{1}, x_{2}, \ldots, x_{N}\right]^{T}, \quad \mathbf{E}=\left[E_{1}, E_{2}, \ldots, E_{N}\right]^{T}$ and $\mathbf{p}=$ $\left[p_{1}, p_{2}, \ldots, p_{N}\right]^{T}$.

On the other hand, the PG's target is to decide on its price $p_{i}$ based on the offered energy $x_{i}$ by EU $i$ so as to minimize the total cost of purchase $\tilde{J}(\mathbf{p})=\sum_{i} J\left(p_{i}\right)$. Here, $J\left(p_{i}\right)$ is the individual cost including the cost of purchasing with price $p_{i}$ and other associated costs. For $J\left(p_{i}\right)$, we consider a convex cost function

$$
J\left(p_{i}\right)=x_{i} p_{i}{ }^{2}+a_{i} p_{i}+b_{i} ; a_{i}, b_{i}>0 ; \forall i \in \mathcal{N},
$$

which is comparable to the practical cost function of some utility companies [3]. In (5), the first term captures the cost of purchasing energy, whereas the associated $\operatorname{costs}^{1}$ are reflected in the last two terms. In (5), $J$ possesses the following properties: i) $J$ increases with the increase of $p_{i}$. That is $J\left(\hat{p}_{i}\right)<J\left(\dot{p}_{i}\right), \quad \forall \hat{p}_{i}<\dot{p}_{i}$; and ii) $J$ is strictly convex. Thus, for $\hat{p}_{i}, \dot{p}_{i} \geq 0, \forall i$ and any real number $0<\gamma<1$, $J\left(\gamma \hat{p}_{i}+(1-\gamma) \hat{p}_{i}\right)<\gamma J\left(\hat{p}_{i}\right)+(1-\gamma) J\left(\hat{p}_{i}\right)$. The objective of the PG is to minimize its total cost, and thus, the net cost

\footnotetext{
${ }^{1}$ Examples include transmission and artificial tariff costs.
} 
function of the PG is

$$
\begin{aligned}
\tilde{J}(\mathbf{p}) & =\sum_{i=1}^{N}\left(x_{i} p_{i}{ }^{2}+a_{i} p_{i}+b_{i}\right) ; \\
\text { s.t., } \sum_{i} p_{i} & =P_{r} ; p^{\min } \leq p_{i} \leq p^{\max } \forall i .
\end{aligned}
$$

As EUs are owned by individual consumers, the PG cannot directly control their decision making processes, and hence, a decentralized control mechanism is required for both EUs and the PG to decide on $x_{i}$ and $p_{i}$ by optimizing their respective utility and cost functions in (4) and (6).

\section{B. Formulation of the game}

The PG and EUs interact with each other to decide on their energy trading parameters $p_{i}$ and $x_{i}$, and we propose an $\mathrm{NSG}^{2}$ $\Lambda$ to capture this interaction. In this game, the PG is the leader who decides on the price $p_{i}$ for the amount of energy offered by the EU $i$, and each EU $i$ is a follower who agrees on $x_{i}$ to be offered to the PG in response to $p_{i}$ by playing a generalized Nash game (GNG) [11] with other followers in the SG. Hence, the proposed NSG can be defined by its strategic form as

$$
\Lambda=\left\{(\mathcal{N} \cup\{\mathrm{PG}\}),\left\{\mathbf{X}_{i}\right\}_{i \in \mathcal{N}}, \hat{U}, \tilde{J}, \mathbf{p}\right\},
$$

where $(\mathcal{N} \cup\{\mathrm{PG}\})$ is the set of all players in the game, and $\left\{\mathbf{X}_{i}\right\}_{i \in \mathcal{N}}$ is the vector of strategies of EU $i$ satisfying (1).

In this game, an EU's decision is affected by the strategies of other EUs through (1), and thus, the GNG amongst EUs, to decide on $x_{i} \forall i$, is a jointly convex generalized Nash equilibrium problem (GNEP) [11], whose solution is a generalized Nash equilibrium (GNE). The proposed game is played in two steps. In the first step, the game is initiated with the announcement of the price $p_{i}=p, \forall i$ satisfying (2) by the PG, upon which the EUs play a GNEP to decide on the GNE energy set $\mathbf{x}$ they wish to sell to the PG. The PG receives the offered energy $x$ and thereby obtains some insight into the capacity ${ }^{3}$ of each EU $i$. In the second step, the PG optimizes its price vector to $\mathbf{p}^{*}=\left[p_{1}{ }^{*}, \ldots, p_{N}{ }^{*}\right]^{T}$, by a constraint optimization technique for the offered $\mathrm{x}$. Thereafter, the EUs again decide on their GNE energy vector $\mathbf{x}^{*}=\left[x_{1}{ }^{*}, \ldots, x_{N}{ }^{*}\right]^{T}$ in response to $\mathbf{p}^{*}$ and the proposed NSG reaches its noncooperative Stackelberg equilibrium (NSE).

Definition 1: Consider the NSG $\Lambda$ in (7), in which $\hat{U}$ and $\tilde{J}$ are defined by (4) and (6) respectively. A set of strategies $\left(\mathbf{x}^{*}, \mathbf{p}^{*}\right)$ constitutes the NSE of the game if and only if the strategy set satisfies the following set of inequalities:

$$
\begin{array}{r}
\hat{U}\left(x_{i}{ }^{*}, \mathbf{x}_{-i}{ }^{*}, \mathbf{E}, \mathbf{p}^{*}\right) \geq \hat{U}\left(x_{i}, \mathbf{x}_{-i}{ }^{*}, \mathbf{E}, \mathbf{p}^{*}\right), \\
\forall x_{i} \in \mathbf{x}, \quad \forall i \in \mathcal{N}, \sum_{i} x_{i} \leq E_{\mathrm{PG}},
\end{array}
$$

and

$$
\begin{array}{r}
\tilde{J}\left(p_{i}{ }^{*}, \mathbf{p}_{-i}^{*}\right) \leq \tilde{J}\left(p_{i}, \mathbf{p}_{-i}^{*}\right) \\
\forall i \in \mathcal{N}, \forall p_{i} \in \mathbf{p}, p^{\min } \leq p_{i} \leq p^{\max }
\end{array}
$$

\footnotetext{
${ }^{2}$ A similar form of game for charging of electric vehicles in smart grid was used in [12].

${ }^{3}$ For a similar price $p$, each EU receives a similar incentive, and thus their offered energies reflect their capacities of supply.
}

Here, $\mathbf{x}_{-i}$ is the energy vector of all EUs in the set $\mathcal{N}$ except EU $i$, and similarly, $\mathbf{p}_{-i}$ is the price vector set by the PG for all EUs in $\mathcal{N}$ excluding $i$.

Therefore, at the NSE, neither any EU nor the PG can increase its utility by deviating from its NSE strategy while all other players in the SG are playing their NSE strategies.

\section{Existence and optimality}

In this section, we investigate the existence of a socially optimal solution which is beneficial for all the consumers in the SG, and thus, suitable for the proposed consumerprioritized energy management scheme. In this regard, first we note that the proposed NSG reaches the NSE as soon as all EUs agree on a GNE energy vector $\mathrm{x}^{*}$ in response to the optimized price vector $\mathbf{p}^{*}$ set by the PG. Due to the strict convexity of (6), there always exists a unique solution [13] for the price vector $\mathbf{p}^{*}$, and thus, the existence of a socially optimal GNE of the followers' GNEP would guarantee the existence of a socially optimal NSE of the proposed NSG.

Theorem 1: There exists a socially optimal GNE solution for the GNEP amongst the EUs in response to the price set by the PG.

Proof: First, we note that the proposed GNEP is a jointly convex GNEP due to the coupled constraint (1), and hence, the GNEP can be formulated as a variational inequality (VI) problem $\operatorname{VI}(\mathbf{X}, \mathbf{F})$ [11], which can be used to determine a vector $\mathbf{x}^{*} \in \mathbf{X} \in \mathbb{R}^{n}$ such that $\left\langle\mathbf{F}\left(\mathbf{x}^{*}\right), \mathbf{x}-\mathbf{x}^{*}\right\rangle \geq 0, \forall x \in \mathbf{X}$. Here, $\mathbf{X}$ is the vector of strategies of all EUs satisfying (1) and, from [11]

$$
\mathbf{F}=-\left(\nabla_{x} U\left(x_{i}, E_{i}, p_{i}\right)\right)_{i=1}^{N} .
$$

The solution of a VI is a variational equilibrium (VE), which is the socially optimal solution among other GNEs [11]. To this end, our main concern it to study the existence of the VE of the GNEP, and thus, check the optimality of the solution. Hereinafter, we will use GNEP and VI interchangeably, and VE to refer to both the VE and GNE of the GNEP. Now, the pseudo-gradient of the joint utility function $\hat{U}$ in (4) is [11]

$$
\mathbf{F}=\left[\begin{array}{c}
x_{1}-E_{1}-p_{1} \\
x_{2}-E_{2}-p_{2} \\
\vdots \\
x_{N}-E_{N}-p_{N}
\end{array}\right],
$$

and the Jacobean of (11), JF, is an identity matrix. Hence, $\mathbf{J F}$ is positive definite on $\mathbf{X}$, and consequently, $\mathbf{F}$ is strictly monotone. Therefore, $\operatorname{VI}(\mathbf{X}, \mathbf{F})$ admits a unique VE [14]. Moreover, due to the joint convexity of the proposed GNEP, the unique VE is also the global unique maximizer of (4) [14], and thus, the existence of a socially optimal VE of the proposed GNEP is proved.

From Theorem 1, we can further conclude that the proposed NSG possesses a socially optimal NSE.

\section{Algorithm}

In this section, we propose a distributed algorithm for the considered NSG to reach the socially optimal NSE. The algorithm is implemented based on the fact that $\mathbf{F}$ is strongly 
monotone, and thus, the slack variables $\mu_{i}=E_{i}-x_{i}+p_{i}, \forall i \in$ $\mathcal{N}$ all possess the same value $\mu_{i}=\mu$ at the VE [14]. We assume that there is limited communication between the PG and EUs via the two-way communications of the SG, and thus, the PG can inform ${ }^{4}$ the EU $i$ if its offered energy $x_{i}$ is beyond the $\mathrm{VE}$ in response to $p_{i}^{*}$ (by checking whether $\left.\mu_{i}=\mu, \forall i\right)$. We use a hyperplane projection method for the VI, which is the simplest solution method for a monotone VI [11], and its solution is always guaranteed to converge to a non-empty VE [11]. Since the PG's optimization problem is strictly convex and has a unique solution, consequently, the proposed algorithm also guarantees that the NSG will reach its socially optimal NSE.

The algorithm is executed in two steps as shown in Algorithm 1. As a hyperplane projection method, we use the S-S method $[11,15]$, which is based on a geometrical interpretation and uses two projections per iteration. Let $x^{l}$ be the current approximation of the solution of $\mathrm{VI}(\mathbf{X}, \mathbf{F})$. In the S-S method, first the projection $r\left(x^{l}\right)=\operatorname{Prj}_{\mathbf{X}}\left(x^{l}-F\left(x^{l}\right)\right)$ is computed ${ }^{5}$, and then a point $z^{l}$ is determined in the line segment between $x^{l}$ and $r\left(x^{l}\right)$, using an Armijo-type search [11]. Then, $x^{l}$ is separated from the other solution $x^{*}$ of the problem via the hyperplane $\delta M^{l}=\left\{x \in \mathbb{R}^{n} \mid\left\langle F\left(z^{l}\right), x-z^{l}\right\rangle=0\right\}$. Now, as soon as the hyperplane is constructed, $x^{l+1}$ is computed in the next iteration onto the feasible set of $\mathbf{X}$ with the hyperspace $\delta M^{l}=\left\{x \in \mathbb{R}^{n} \mid\left\langle F\left(z^{l}\right), x-z^{l}\right\rangle \leq 0\right\}$ which contains the solution set [15]. The details of the S-S method can be found in [15].

\section{NUMERICAL EXPERIMENTS}

We simulate the proposed energy management scheme by considering a number of different EUs, where each EU represents 20 similar energy consumers connected via an aggregator. The available SE of each EU is assumed to be a uniformly distributed random variable in the range of $[64,240]$ kilowatt hour $(\mathrm{kWh})$, and thus covers both the lowest battery capacity of a group of solar panels $(3.6 \mathrm{kWh}$ per panel) and the highest battery capacity of a wind turbine group (12.25 kWh per turbine). The total price per unit energy is assumed to be 175 US cents ${ }^{6}$, and $\left(p^{\min }, p^{\max }\right)=(8.45,175)$ cents, unless stated otherwise. All the statistical results are averaged over random values of the EUs' capacities using 100 independent simulation run

In Fig. 1, the convergence of the utility achieved by each EU from selling its energy is shown to reach the NSE. We consider that 5 EUs are connected to the PG, and as shown in the figure, the utility achieved by each EU reaches its NSE after the $7^{\text {th }}$ iteration. Importantly, the EU with a larger amount of SE has a higher utility, which is due to the fact that it can sell more energy to the PG, and hence, it is being paid more. Consequently, its utility is larger.

Next, we demonstrate the effectiveness of the proposed scheme by comparing its performance with a standard feed-in

\footnotetext{
${ }^{4}$ This can be done, for example, by using a single bit.

${ }^{5} \operatorname{Prj}_{\mathbf{X}}(k)=\arg \min \{\|\omega-k\|, \omega \in \mathbf{X}\}, \omega \in \mathbb{R}^{n}$

${ }^{6}$ For 5 EUs, the average price per unit energy is 35 cents/kWh [16].
}
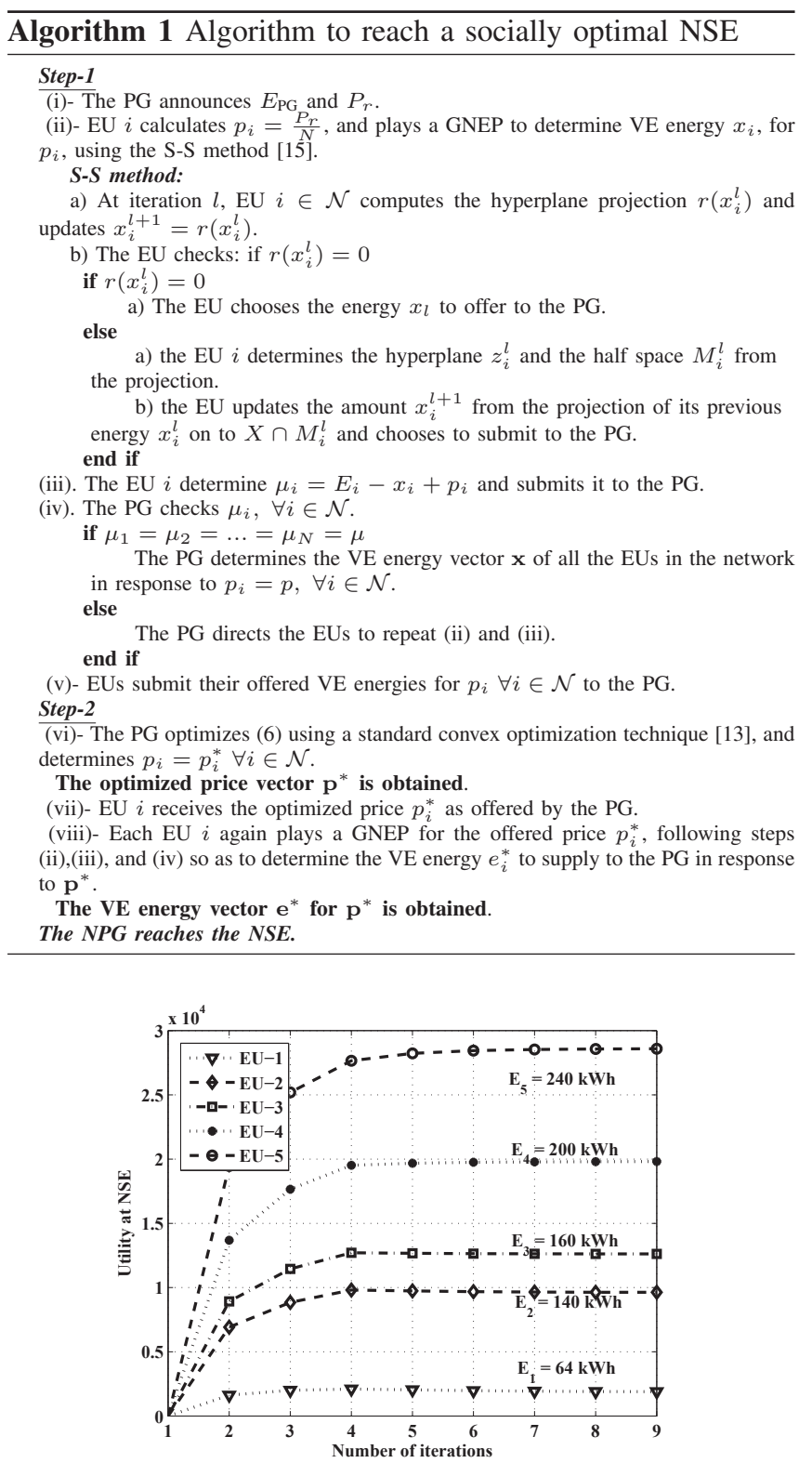

Fig. 1: Convergence of the utility to NSE.

tariff scheme (FIT) [16]. We note that an FIT is an incentive based energy trading scheme which is designed to increase the use of renewable energy systems providing power to the main grid when it is required. A higher tariff is paid to the energy producers to encourage them to take part in energy trading. For comparison, here we assume that the contract between the EUs and the PG is such that the EUs are capable of providing the $\mathrm{PG}$ with the required energy with a tariff of 60 cents per kWh [16]. In Fig. 2, we show the performance comparison between the proposed and FIT schemes for the average achieved utility per EU as the number of EUs varies in the SG. As shown in this figure, an increase in the number of EUs subsequently increases the freedom of the PG to buy its energy from more EUs, and hence, the amount of energy sold 


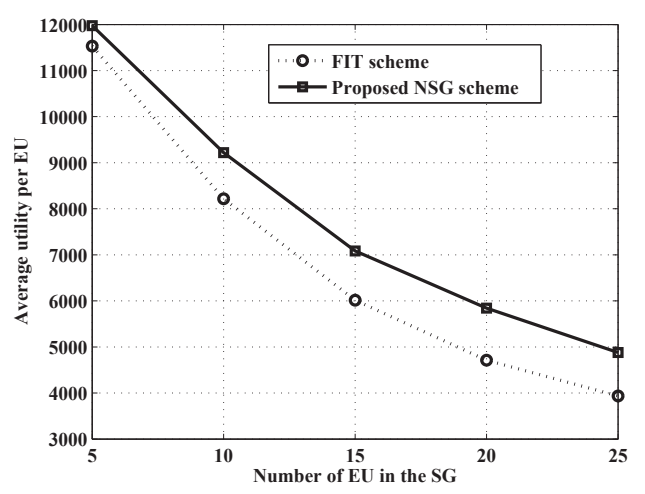

Fig. 2: Effect of the network size on the average utility per EU.

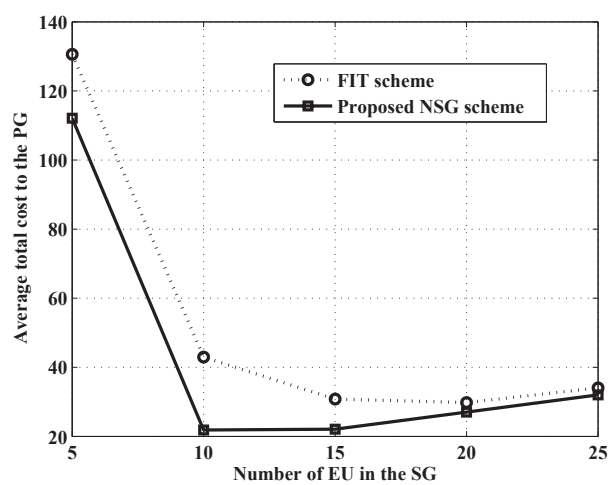

Fig. 3: Effect of the network size on the average total cost of the PG.

by each EU decreases. As a result, average utility decreases for both the schemes. However, the proposed NSG, due to its capability of choosing an optimal energy for maximizing the EUs' benefits, always shows a considerable improved performance over the FIT scheme in terms of average utility per EU. As seen in Fig. 2, the utility per EU for the proposed NSG is 1.5 times, on average, better than the utility achieved by an FIT scheme.

The effect of the number of EUs on the average total cost to the PG is shown in Fig. 3 for both the NSG and the FIT schemes for the same total price per unit of energy $P_{r}$. For a fixed $E_{\mathrm{PG}}$, increasing the number of EUs from 5 to 15 allows the PG to buy its energy from more EUs, and thus, enables the PG to pay a cheaper rate. Consequently, the total cost incurred by the PG decreases. However, to keep all the EUs participating, the PG needs to pay the minimum mandatory price $p^{\min }$ to each EU. Thus, as the number of EUs increases from 20 to 25 , the total cost to the PG increases due to the mandatory payment to a large number of EUs. Fig. 3 shows that the proposed scheme has significantly lower total cost to the PG at small network sizes, e.g., for 10 EUs the average total cost for the proposed scheme is half the total cost incurred by the FIT scheme. However, as the network size increases, the average total cost for the proposed NSG becomes closer to the FIT scheme. In fact, as the network size increases, the
PG needs to optimize its price for a large number of EUs while maintaining the minimum payment. Hence, due to the constraint (2), a large number of EUs causes the PG to choose a price close to its minimum payment and consequently, the total cost for the proposed NSG becomes closer to that of the FIT scheme.

\section{CONCLUSION}

In this paper, we have studied a demand-supply balance technique by prioritizing consumer benefits, and have proposed a Stackelberg game which leads to a socially optimal Stackelberg equilibrium. We have shown that the proposed scheme maximizes the utility of the end users at the solution of the game, and at the same time keeps the total cost to the power grid to a minimum. We have studied the properties of the game analytically including the existence and the social optimality of the studied scenario. The effectiveness of the scheme has been demonstrated with considerable performance improvement when compared to a standard feed-in tariff scheme.

\section{REFERENCES}

[1] X. Fang, S. Misra, G. Xue, and D. Yang, "Smart grid - the new and improved power grid: A survey," IEEE Communications Surveys Tutorials, vol. PP, no. 99, pp. $1-37,2011$.

[2] T. Başar and G. L. Olsder, Dynamic Noncooperative Game Theory. Philadelphia, PA: SIAM Series in Classics in Applied Mathmatics, Jan. 1999.

[3] A. Mohsenian-Rad, V. Wong, J. Jatskevich, R. Schober, and A. LeonGarcia, "Autonomous demand-side management based on gametheoretic energy consumption scheduling for the future smart grid," IEEE Transactions on Smart Grid, vol. 1, no. 3, pp. 320 -331, Dec. 2010.

[4] R. Walawalkar, S. Fernands, N. Thakur, and K. R. Chevva, "Evolution and current status of demand response (DR) in electricity markets: Insight from PJM and NYISO," Energy Journal, vol. 35, no. 4, pp. 1553-1560, Apr. 2010.

[5] C. Wu, H. Mohsenian-Rad, and J. Huang, "Vehicle-to-aggregator interaction game," IEEE Transactions on Smart Grid, vol. 3, no. 1, pp. 434 -442, Mar. 2012.

[6] W.-H. Liu, K. Liu, and D. Pearson, "Consumer-centric smart grid," in Proc. of the IEEE PES Innovative Smart Grid Technologies, Anaheim, CA, Jan. 2011, pp. $1-6$.

[7] S. Han, S. Han, and K. Sezaki, "Development of an optimal vehicle-togrid aggregator for frequency regulation," IEEE Transactions on Smart Grid, vol. 1, no. 1, pp. $65-72$, Jun. 2010.

[8] P. W. Farris, N. T. Bendle, P. E. Pfeifer, and D. J. Reibstein, Marketing Metrics: The Definitive Guide to Measuring Marketing Performance. Upper Saddle River, NJ, USA: Pearson Prentice Hall., 2010.

[9] Z. Yun, Z. Quan, S. Caixin, L. Shaolan, L. Yuming, and S. Yang, "RBF neural network and ANFIS-based short-term load forecasting approach in real-time price environment," IEEE Transactions on Power Systems, vol. 23, no. 3, pp. 853-858, Aug. 2008.

[10] P. Samadi, A. Mohsenian-Rad, R. Schober, V. Wong, and J. Jatskevich, "Optimal real-time pricing algorithm based on utility maximization for smart grid," in Proc. of the First IEEE International Conference on Smart Grid Communications, Gaithersburg, MD, Oct. 2010, pp. $415-$ Smart

[11] D. Arganda, B. Panicucci, and M. Passacantando, "A game theoretic formulation of the service provisioning problem in cloud system," in Proc. of the International World Wide Web Conference, Hyderabad, India, Apr. 2011, pp. 177 - 186.

[12] W. Tushar, W. Saad, H. V. Poor, and D. B. Smith, "Economics of electric vehicle charging: A game theoretic approach," IEEE Transactions on Smart Grid, vol. 3, no. 4, pp. 1767 - 1778, Dec. 2012.

[13] S. Boyd and L. Vandenberghe, Convex Optimization. New York, USA: Cambridge University Press, Sep. 2004.

[14] F. Facchinei and C. Kanzow, "Generalized Nash equilibrium problems," $4 O R$, vol. 5, pp. $173-210$, Mar. 2007.

[15] M. V. Solodov and B. S. Svaiter, "A new projection method for variational inequality problems," SIAM Journal on Control and Optimization, vol. 37, no. 3, pp. 765-776, 1999.

[16] S. Choice, "Which electricity retailer is giving the best solar feed-in tariff," website, 2012, http://www.solarchoice.net.au/blog/ which-electricity-retailer-is-giving-the-best-solar-feed-in-tariff/. 